\title{
Design and Application of Cloud-based Operation and Maintenance Platform for Industry $\mathbf{4 . 0}$
}

\author{
Jiqing Cao ${ }^{1, a}$, Shuhai Zhang ${ }^{2, b}$ \\ ${ }^{1}$ Department of Information Engineering, Suzhou Industrial Park Institute of Service Outsourcing \\ Suzhou, 213123, China \\ ${ }^{2}$ Department of IT Service, Bosch Automotive Products (Suzhou) Co., Ltd.Suzhou, 215123, China \\ aemail: grinner@21.com, bemail:zhangsh @tom.com
}

Keywords: Industry 4.0; Cloud Computing; Operation and Maintenance; Big Data; DevOps; ITIL

\begin{abstract}
Industry 4.0 has high demands on the system performance, capacity, continuity and security and the traditional IT operations cannot meet the requirements any more. In order to adapt to the new requirements of Industry 4.0, this paper designs the cloud platform architecture for the IT operation and maintenance based on the DEVOPS principle with the new technologies of Cloud Computing, Big Data, Automation, Virtual Reality, RFID, OPC UA and ESB etc. The functions of the platform include achieving a rapid and automatic recovery after a system error, predicting failures and security situation, solving the accessing and processing problems of the heterogeneous equipment and systems involved in Industry 4.0 vertical integration, horizontal integration and end to end integration. The successful practice of the operation and maintenance platform solution in the enterprise proves it can meet the requirements of Industry 4.0 business and technologies, and greatly improve the efficiency of IT operations.
\end{abstract}

\section{Introduction}

The key support of Industry 4.0 is intelligence and information, and intelligence is based on the large amounts of data generated by information [1]. Industry 4.0 has a very high demand on performance, capacity, continuity and security. In the traditional mode of operation and maintenance, complex range of intelligent devices and virtual IT resources make the operation and maintenance work more complex, staff responsibilities in different departments is unclear, maintenance works are mainly "passive service", proactive prevention services is lacked, while burdensome business and approval process leads to inefficiencies, and some of the risks and faults can only be treated afterwards and cannot be predicted in advance. So, the traditional operation and maintenance management cannot manage the complexity of the new Industry 4.0 environment any more, we must start to establish the new mode for operation and maintenance of Industry 4.0[2].

Following the design principles and standards of DEVOPS, Cloud Computing, Automation and Visualization, the paper designed a Cloud Operation and Maintenance Platform, which achieved various functions such as the close collaboration among the different functional departments, the standardization, virtualization, prediction, automation, visualization, industrial network security, mobile operation and maintenance and distributed heterogeneous processing of the IT service management. It can effectively reduce the costs of operation and improve the service levels and efficiency for the IT service and management.

\section{Challenge to Traditional Operation and Maintenance}

Germany raised Industry 4.0 is divided into three integration systems, namely, vertically integration, horizontal integration, and end to end integration. These integrations involves workshops, factories, supply chains and the different industry chains, including PLC (Programmable Logic Controller), PCS(process control system), MES(Manufacturing Execution Systems), ERP (Enterprise Resource Planning) and the across-organization and transregional industrial internet system. At present, there is not yet a final standard in the data standard, but the 
technical architecture of Industry 4.0 is confirmed, including Cloud Computing, Big Data, Mobile Internet, IoT (Internet of Things), Artificial Intelligence, Virtual Reality and industrial network safety. [3]

The traditional ITIL-based operation and maintenance focus on managing IT issues through "IT service standardization" and the split among the transactions is very obvious. What's more, the ITIL-based operation and maintenance have not considered the affecting factors of the new technologies such as virtualization and Big Data etc., so that it is unable to meet the new requirements of Industry 4.0 [4]. In short, the challenges to the traditional operation and maintenance are [5]:

1) Be able to achieve the effective monitoring and management for the infrastructures, systems and virtual resources;

2) Be able to quickly and automatically locate and process systems failures and potential risks;

3) Be able to achieve the collection, storage, analysis of the massive data and support the decisions.

4) Be able to proactively avoid problems, to achieve self-management of the equipments and systems;

5) Be able to achieve the mobile inspection and remote maintenance;

6) Be able to ensure the high system availability and achieve fast, automated deployment;

7) Be able to meet the production and network security of the Industry 4.0..

\section{Design of Cloud-based Operation and Maintenance Platform}

\subsection{Industry Standards and Practices}

ITIL, IT Infrastructure Library, is currently the international de facto standard for IT operation and maintenance. It includes four stages of Services Strategy, Service Design, Service Transition, Service Operation and the Continuous Improvement of Services according to the life cycle of IT services. Among them, the service operations describes the system operation and maintenance including Event Management, Incident Management, Problem Management, Configuration Management, Change Management, Release Management, and Security Management Processes, etc.

ISO27001 specifies the Information Security Management System and the requirements for information security control. It is the foundation of information security management system implementation and evaluation of the organization. The standards include more than 120 security controls to help an organization to identify their impacts on information security practices, including security policy, information security organization, human resources security, physical and environmental security, communications and operational security, access control, system acquisition, information security incident management, business continuity management and so on. [6]

DevOps represents the latest agile, lean and automation thinking advocated by the current operation and maintenance management. It is the new mode of operation and maintenance proposed under the new technologies of Cloud Computing and the background of automated operation and maintenance, which stresses the idea of automating and continuing everything for operation and maintenance. With the DEVOPS thinking, it must require an integrated platform to schedule these transactions flow together to achieve an automated and efficient operation and maintenance. [7].

3.2 Design Principles and Objectives

Based on the characteristics of Industry 4.0, the operation and maintenance platform established the design principles that it follows ITIL and ISO27001 standards, uses the technologies of Cloud Computing, Big Data, Virtual Reality, OPC UA (OLE for Process Control Unified Architecture) and ESB (Enterprise Service Bus) etc. to achieve cloud-based, automation, visualization and the security of the operation and maintenance of Industry 4.0.

The specific purposes of the design of the cloud-based operation and maintenance platform are: To establish an automated "Big Operation and Maintenance" platform based on DEVOPS thinking, so as to coordinate the teamwork of different departments; To achieve the high availability of the operation and maintenance by constructing the operation and maintenance platform architecture on 
the cloud platform; To build the physical and operational topology relationship for the business, assets and systems, and achieve to optimize production and failure prediction with the technologies of Big Data and Virtual Reality etc.; To automate all operation and maintenance processes by following ITIL standard and automation technology; To realize the data acquisition from the intelligent devices, as well as interconnection of different distributed, heterogeneous systems by the technologies of OPC UA and ESB etc.

\subsection{Cloud-based Platform Architecture Design}

Based on the design principles and objectives above, the architecture of the Cloud-based Operation and Maintenance Platform for Industrial 4.0 (see Figure 1) includes four layers, which are IaaS Layer (Infrastructure as a Service), PaaS Layer (Platform as a Service), SaaS Layer (Software as a Service), as well as the Unified Interface Layer. IaaS Layer is also known as resource management and data acquisition layer, covering the management of various of resources, infrastructure and basic services. PaaS layer, also known as platform management and data processing layer, it provides the core platform functions of Cloud Computing, Visualization, Big Data and Automation based on the corresponding technology platforms. SaaS Layer, also known as operation and maintenance services layer, which achieves the core business of operation and maintenance, such as Infrastructure Management Cloud, Data Management Cloud, and Monitoring and Alarms Cloud and so on. The Unified Interface Layer is the interface of the cloud platform to all systems and users of the Industry 4.0. [8]

\begin{tabular}{|c|c|c|}
\hline $\begin{array}{l}\text { Unified Interface } \\
\text { Layer }\end{array}$ & $\begin{array}{l}\text { Unified Interface to } \\
\text { System, User and Presentation }\end{array}$ & \multirow{4}{*}{$\begin{array}{l}\text { Common Tech: } \\
\text { Cloud Platform } \\
\text { Big Data } \\
\text { Virtual Reality } \\
\text { Smart Position } \\
\text { RFID } \\
\text { ESB } \\
\text { OPC UA } \\
\text { ProfiNet }\end{array}$} \\
\hline $\begin{array}{l}\text { Operation\& } \\
\text { Maintenance. } \\
\text { Service Layer } \\
\quad \text { ( SaaS })\end{array}$ & $\begin{array}{l}\text { Cloud Set of Infra. Mgt, Auto. } \\
\text { Process Mgt, Monitoring, Data } \\
\text { Driven Service, Production } \\
\text { Optimization }\end{array}$ & \\
\hline $\begin{array}{l}\text { Platform } \\
\text { Mgt .Layer } \\
\text { (PaaS) }\end{array}$ & $\begin{array}{l}\text { CMDB } \\
\text { Platform for Big Data, Cloud } \\
\text { Computing, Cloud Storage, Auto } \\
\text { Engine; }\end{array}$ & \\
\hline $\begin{array}{l}\text { Data Acquisition } \\
\text { Layer } \\
\text { (IaaS) }\end{array}$ & $\begin{array}{l}\text { Data Acquisition through } \\
\text { UPC UA, Equipment, Sensor, } \\
\text { PLC, MES }\end{array}$ & \\
\hline
\end{tabular}

Fig.1. Industry 4.0 Cloud-based Operation and Maintenance Platform Architecture

Meanwhile, the operation and maintenance platform is built on a number of key common technologies and functional modules, including Cloud Computing, Big Data, Virtual Reality, Smart Positioning, RFID (Radio Frequency Identification, RFID), ESB, OPC UA, ProfiNet and Industrial Network Security to support the different functional modules on the four layers of the cloud-based platform. They were introduced as follows:

\section{1) Resource Management and Data Acqusition Layer}

This layer achieves the data collection from the remote, distributed and various heterogeneous devices and production systems through the technologies of industrial Ethernet, ProfiNet, OPC UA and ESB, and the interconnection among the different devices and systems of the vertical integration, horizontal integration and end-to-end integration of Industry 4.0. [9]

2) Platform Management and Data Processing Layer

Based on the technology platforms of Cloud Computing, Virtual Reality, Big Data, GIS (Geographic Information System) and Automation Engine, this layer provides the core functions of Distributed Cloud Storage, Visualization, Production Optimization, Smart Positioning, Automation 
Process Management and Data Configuration Management to the modules of the Operation and Maintenance Service Layer through a unified interface.

The non-relational data are stored in the Distributed Cloud Storage System for the real-time big data processing. For relational data, such as the data described the relationships topology of physical equipments and business processes, they will be maintained centrally in the Configuration Management Database (CMDB), which is the core system for the interconnection among different modules. [10]

3) Operation and Maintenance Service Layer

This layer includes the cloud sets of the automated operation and maintenance application processes based on ITIL best practices. It is a functional core of the Operation and Maintenance Platform, including Infrastructure Management Cloud, Automated Operation and Maintenance Process Management Cloud, Monitoring and Alarm Management Cloud, Data-driven Service Cloud and Production Optimization Cloud etc. Meanwhile, these application cloud set are interdependent and work together to improve the quality and efficiency of the entire operation and maintenance works. Specific features of this layer are described below:

a) Infrastructure Management Cloud

Based on the technologies of CMDB, Virtual Reality, GIS and RFID etc., this Module achieves the visual management on the various resources and equipment of the Industry 4.0 Automation Systems and IT Systems, such as asset identification, network topology and positioning. Coordinating with the Automation Module and Data Driven Service Module, it can also achieve the functions of automatic repair, mobile inspection and pre-warning, etc for the infrastructure.

b ) Automated Process Management Cloud

Taking into account the affect on the process by the characteristics of these technologies such as Virtualization, Big Data, and Automation, this Module designs the uniform and new automated operation and maintenance processes, the main features include Event Management, Incident Management, Configuration Management and Release Management, etc. When there are failures in the system, it can achieve automated alarming, positioning, automated deployment and even the automated replacement for the smart devices. For the incidents needing manually treatment, they will enter the automated work order process and be handled by the corresponding owner defined. It achieves the standardization, virtualization and automation of IT service management, and improves the efficiency of the operation and maintenance works.

c) Monitoring and Alarming Management Cloud

This module achieves the intelligent control, including monitoring of automation systems, IT systems and the video surveillance of production environments, which dynamically shows the operational status of equipment and resources, trends of power consumption and status of sensors. Through real-time and historical data analysis, this module achieves the pro-warning of the system failures and smart positioning, so that the service quality and the resource consumption of hotspots business services are visible and measurable. [11]

d) Data Driven Service Cloud

The module is focused on analysis and processing for the data from the Data Acquisition Layer, and is the data driver core for the functional modules on each layer of the platform. Through the analysis of the real-time data with Big Data technologies, this module can support to achieve the real-time incident predictive maintenance, and the early warning for the industrial network security, etc. The common business data will be stored in the CMDB, which can be used to analyze the current problems and risks with Six Sigma techniques, further improve the quality of production processes and products, and achieve the lean and agile production management. [12]

e) Production Optimization Cloud

Self-aware and flexible production mode of many varieties, small batch and fast response is important features of Industry 4.0. This module analyze the data from equipment and its surrounding environment using Big Data technologies, processes real-timely and feedbacks to the device controller which will automatically adjust according to changes in product processing situation, so as to achieve self-aware, automatic scheduling, load balancing and production 
optimization of the equipment. [13]

4) Unified Interface Layer

This layer is the interface of external service provider for the entire platform, including a variety of interfaces to external systems, the user interface and presentation interface. The External System Interface achieves the interface function with systems of vertical integration, horizontal integration, and end-to-end integration of Industry 4.0. The User Interface achieves the access interface function for the users to operate on the various applications and operation and maintenance services on the platform. The Presentation Interface presents a variety of professional reports, visualization models and alarm signals for the operation and maintenance engineer to make scientific decisions upon these rich reports.

The layer can display the relevant screens on the computer, mobile phone, tablet, and wearable devices in a uniform, intuitive presentation, and allow users to control the operation of devices and systems anytime, anywhere. It provides very efficient support for emergencies and emergency events, and enables mobile operation and maintenance management. [14]

5) Industry Network Security

The security of Industry 4.0 not only directly affect the production, life and property safety of employees and enterprises, but also may be involved in the Industry and even national level security. It is a common module for the security management for all the layers and is designed by reference of ISO27001. In addition to the IT level of general information security, this module covers different aspects of the automation systems, IT systems and managements, and achieves the functions of system boundary protection, protocol testing, equipment protection and remote maintenance etc., summarized below:

a) Security Situation Analysis and Early Warning.

The function uses Big Data technologies and professional tools to realize information security early warning systems, analyze equipment and systems logs and automatically assess, predict the security, stability and service life of equipment, as well as respond to unexpected incidents of the network. Coordinate with visualization techniques, it can efficiently and timely draw the map of security situation, enable decision makers to make a reliable assessment and treatment for the security situation. [15]

b) Security Event Management.

The security events (such as hacking, viruses, unauthorized access, security between the illegal operation of different systems to exchange information, network isolation) identified through different channels such as process monitor, audit and industrial firewalls, etc. are treated by automated manner preferentially. For those cannot be completed by the automation will be treated by the Event Management Process of the platform.

c) Virtual Resources Management

With virtualization and migration technologies of Cloud Computing, the emergency response mechanism is established. The operation and maintenance engineer only need some clicks of the mouse in the cloud application, the virtual resources such as virtual firewall, virtual computer and virtual networks can be repaired, which ensures the continuity and availability of the business. [16]

d) Security Personnel Position Management.

RFID technology can be used to locate the specific location of the security personnel which is very valuable for the operation and maintenance situation of across the engine room, across the workshop and large-scale inter-organizational systems. Once danger, managers can use this system to direct security work. [17]

e) Physical and Environmental Security.

This function focus on equipment safety and service life, prevent loss of assets, theft or activities threatening the production, equipment, personnel and business. Once unexpected events occur, video security monitoring function can be coordinated with other subsystems like visualization model etc. to manage the emergences to ensure the safety of the assets of the equipment.

f) Data Security. 
Proprietary technology and tools meeting the industry network security are introduced to achieve the data security, such as industrial-level firewall, OPC deep packet detection technology, CIFS (Common Internet File System) antivirus technology, and TLS (Transport Layer Security Protocol) encryption technology etc.

3.4 Key Technologies for the Platform

1) Cloud Computing

With virtualization technology, cloud computing assigns the hardware resources as shared resources to the system as on-demand service, which improves resource utilization. This technology can achieve rapid deployment with the dynamic migration function of the virtual machines, so as to ensure continuity of the business. To save the data in the distributed cloud-based storage system can meet the access needs of Industry 4.0 mass data. To build the operation and maintenance platform on the cloud computing platform can not only enhance the quality of IT services, reduce IT operation and maintenance costs, but also improve the controllability of IT systems. [18]

2) Big Data

Big Data technologies can collect, storage, measure and analyze the massive data of the intelligent device, production and logs real-timely, and achieve the self-adaption, load balancing, incident prediction, early warning for security risks, production optimization and quality management capabilities of the equipment and systems, which provide effective data support and intelligent decision support for the Industry 4.0.

3) Virtual Reality

In support of Big Data, Virtual Reality can show global monitoring data with visual model, breakthrough the across-geographical limitation, and help the operation and maintenance personnel to understand and master the details of monitoring situation of the overall resources. Coordinating with automation and smart positioning technologies, it can achieve inspection of the facilities and IT systems, positioning of the failure or spare parts, automated deployment of the application, so that everyone understand consistently, implement consistently and result consistently.

4) RFID

RFID, also known as radio frequency identification, is a wireless communications technology. RFID technology can connect goods and IT networks together to achieve intelligent identification and management. The main functions of RFID include cargo tracking, automatic information collection, real-time monitoring of production data, quality tracking and automated production etc.

\section{5) ESB}

ESB, namely enterprise service bus, is the technology of the combination of the traditional technologies of middleware, XML and Web services etc. ESB has changed the traditional software architecture that allows different applications coordinated, enables communication and integration between different services. ESB provides a series of standard interfaces to solve the interoperability and interconnection problems for systems of vertical integration, horizontal integration and end-to-end integration of Industry 4.0 systems. [19]

6) OPC UA

OPC UA is the interoperability standards for secure data exchange for automation control industry. It achieves not only the information integration of the equipment data acquisition, and equipment interoperability, but also achieves the information integration of equipment to SCADA (Supervisory Control and Data Acquisition), SCADA to MES, equipment and the MES. [20]

7) Industrial Ethernet /ProfiNet

Industrial Ethernet is the Ethernet technology used in industrial control field. In order to apply to the harsh industrial environments and ensure the safe and reliable industrial applications, industrial Ethernet technology has the advantages of inexpensive, reliable, high-speed communications, widely used and mature support technology and so on.

ProfiNet is a new generation of automation bus standard based on Industrial Ethernet. It provides a complete network solutions for automation communications, and, as a cross-vendor technology, it is fully compatible with the Industrial Ethernet and existing fieldbus (e.g., ProfiBus) technology to 
protect existing investments.

\section{Implementation of the Platform}

As a pioneer of Industry 4.0, MOTO Automotive Products (Suzhou) Company is a representative of auto parts manufacturers. The company's flexible production line of CNC (Computerized Numerical Control) equipment, robots, materials and auxiliary equipment are intelligent-based and interconnected, each production line deploys a production line console subsystem, and background shares the production line server. The business process, material and personnel information, and production data are stored in the server side. After years of information construction, MOTO has successfully implemented ERP, PDM (Product Data Management), MES and other systems in its subordinate enterprises, these existing systems have accumulated a lot of historical business data.

During its construction of Industry 4.0, MOTO designs its operation and maintenance system based on the cloud-based platform architecture discussed in this paper. They deploy the ESB based on WebLogic Server to connect the different IT systems in the form of Web Services middleware, so that the existing systems continue to be retained; They use ProfiNet to achieve the interconnection between different devices and data collection; They use RFID to achieve the material tracking during production and logistics processes and automatic inventory of the assets; They use cloud storage to achieve the storage of massive production data; They use Big Data technologies to achieve the incident and risk prediction and real-time monitoring of network and equipment; They use Virtual Reality and Smart Positioning technology to achieve the visualization and location management for logistics, factories, workshops, production lines, IT infrastructure and equipment. By introducing the operation and maintenance functions on the mobile devices such as phone, tablet, and wearable devices, they achieve the mobile operation and maintenance management of production lines and logistics system.

Mainly based on ISO27001, MOTO implement the security from all aspects of infrastructure, equipment, resources, networks, systems, data, and management for the industrial network security. With the different security technologies and measures such as Big Data, Security Equipment, Data Encryption Device, Application Authentication and Safety Management System, they establish the security framework for the operation and maintenance programs to ensure the company's safety and security.

The success practice of the operation and maintenance platform in MOTO proved that it meets the requirements of Industry 4.0, which greatly improved the efficiency of IT operations, so that it can be the reference guide for planning, designing, implementation and management for the operation and maintenance of Industry 4.0 corporate.

\section{Conclusion}

First in the operation and maintenance, this paper designs the platform architecture as the specific requirements for Industry 4.0. The design follows the lean and agile principle of DEVOPS, with the latest technologies of automation, OPC UA, ProfiNet, Cloud Computing, Big Data, Virtual Reality, RFID and ESB etc., it can solve the difficulties and challenges the traditional operation and maintenance faced of Industry 4.0, improve the efficiency of operation and maintenance, and ensure the security of Industry 4.0 network and production.

At present, there is still no operation and maintenance platform released for Industry 4.0 domestic and foreign, so this study has obvious relevance and forward-looking. With the gradual promotion and more and more enterprises to implement Industry 4.0, the results of this study will show its value much more.

\section{Acknowledgment}

In this paper, the research was sponsored by the Service Outsourcing Foundation of Suzhou Industrial Park Institute of Service Outsourcing (Project No. KY-XJY02). 


\section{References}

[1] ZHANG Shu. The Industry 4.0 and Intelligent Manufacturing[J]. MACHINE DESIGN AND MANUFACTURING ENGINEERING.2014, (8)

[2] Cao Jian. Analysis of IT operation based on Virtual background [J]. Information \& Communications, 2015(8):154-155

[3] DKE/DIN, The German Standardization Roadmap Industrie 4.0 [R]. VDE ASSOCIATION FOR ELECTRICAL, ELECTRONIC \& INFORMATION TECHNOLOGIES,2014.11

[4] Chen Chunhua, Li Jun.Liang Huan,Yao Wensheng. IT Service Management Implementation Strategy of Resource Pool Environment[J]. Telecommunications Science, 2012(9) :144-146

[5] Wang Lingyun, Lihui, The New Requirement for the equipment management of Industry 4.0[J]. China Plant Engineering 2015(7):2-3

[6]ISO/IEC. ISO27001 . 2005

[7] JAMES ROCHE, Adopting DevOps Practices in Quality Assurance [ J ] .Communications of the ACM, 201311

[8] Niu Xiaoling. Cloud Computing Operation and Maintenance Management System Framework Research[J]. Telecom Network Technologies,2015(2)

[9][20] Ye Xiaogao. Industrial Applications Cutting-edge and Security Analysis of OPC UA [J].Management \& Technology of SME.2015, (33)

[10] FAN Juanjuan LIU Yu LIU Liang WANG Jingyan GAO Mingxing .CMDB Subsystem in railway IT Integrated Operation and Maintenance Management System. Railway Computer Application. 2015, (8)

[11] Liu Ting.Reflections on the operation and maintenance of information technology Based on Industry 4.0[J]Information Technology \& Standardization.2015(6)

[12][15] Li Jie.Industry 4.0 Era: China’s Opportunities are Data and Data Analysis [J].World Science.2014(6)

[13] Li Jie, Cheng Qisen. Predictive Manufacturing under Industry 4.0 Environment[J].World Science. 2014(5)

[14] LIANG Jie. Research and Design of the Visualization of Mobile Online Intelligent Inspection System[J]-wuxian hulian keji.2015, (4)

[16] TANG Jian DONG Jian. Unified Operation and Maintenance of Virtualized Environments[j] Computer Knowledge and Technology

[17] ZHANG Ying-hua [1] PEI Jian-guo [1] CHEN Xing [2] Indoor fire fighter positioning based on FAS system and RFID technology[J] Fire Science and Technology. 2015, (6)

[18] Ning Jun. Affect of Cloud Computing Trend on Network Infrastructure [J].Information and Communications Technologies.2013(1)

[19] DI Jian [1] XIAO Jun [1] WANG Chunxin [2] YANG Youpeng [2] Design of IoT comprehensive service platform based on enterprise service bus[J] .Journal of Computer Applications 